\title{
Burst-mode Yb-doped fiber amplifier system optimized for low-repetition-rate operation
}

\author{
H. Kalaycioğlu, ${ }^{1, *}$ Ö. AkÇaAlan, ${ }^{1}$ S. Yavaș, ${ }^{2,3}$ Y. B. Eldeniz, ${ }^{4,5}$ and F. Ö. Ildday ${ }^{1,6}$ \\ ${ }^{1}$ Department of Physics, Bilkent University, Ankara 06800, Turkey \\ ${ }^{2}$ UNAM-Institute of Materials Science and Nanotechnology, Bilkent University, Ankara 06800, Turkey \\ ${ }^{3}$ FiberLAST, Inc., Ankara 06531, Turkey \\ ${ }^{4}$ Department of Electronics Engineering, Ankara University, Ankara 06100, Turkey \\ ${ }^{5}$ Roketsan, Inc., Ankara 06780, Turkey \\ ${ }^{6}$ Department of Electrical and Electronics Engineering, Bilkent University, Ankara 06800, Turkey \\ ${ }^{*}$ Corresponding author: hamitkal@bilkent.edu.tr
}

Received 28 January 2015; revised 8 March 2015; accepted 8 March 2015; posted 12 March 2015 (Doc. ID 233356); published 27 April 2015

\begin{abstract}
We report on the burst-mode operation of a polarization-maintaining $\mathrm{Yb}$-doped multi-stage all-fiber amplifier capable of generating 10-pulse $100 \mathrm{~ns}$ long bursts of $400 \mu \mathrm{J}$ total energy. The corresponding average energy per pulse is $40 \mu \mathrm{J}$, with a standard deviation of $16 \%$. The $40 \mu \mathrm{J}$ pulses are compressible to a full width at half-maximum of $500 \mathrm{fs}$. The burst repetition rate is set to $1 \mathrm{kHz}$ and the amplifier is synchronously pulse pumped to minimize amplified spontaneous emission (ASE) between the bursts. A special amplifier design has been developed to suppress ASE further at the cost of lower power efficiency. A detailed investigation of gain and ASE suppression performance of the amplifier in relation to pulsed pumping is presented. ( 2015 Optical Society of America
\end{abstract}

OCIS codes: (140.3280) Laser amplifiers; (060.3510) Lasers, fiber; (060.2320) Fiber optics amplifiers and oscillators; (140.3615) Lasers, ytterbium.

http://dx.doi.org/10.1364/JOSAB.32.000900

\section{INTRODUCTION}

There is much interest in fiber amplification of ultrashort pulses, which offers practical advantages such as low-cost, highly robust operation and high-gain amplification [1- $1-\underline{4}]$. These aspects are particularly important for material [5] and tissue [6] processing, in industrial, medical, and scientific settings, outside the laser research laboratory. Despite tremendous technical improvements, particularly in terms of pulse energy and average power, the essentials of laser material processing have changed little: The overwhelming majority of the fibers, as well as solid state lasers, rely on a train of identical pulses with constant temporal spacing, which is not necessarily ideal.

Lapczyna et al. have demonstrated a powerful alternative, where the laser amplifier produces a group of a limited number of high-repetition rate pulses, called a burst, which itself is repeated at a much lower repetition rate [7]. For a long time, burst-mode operation has been confined to diverse, but niche applications, including laser systems in accelerators [줄] $]$, combustion diagnostics [10], flow measurements in aerodynamics [11], Thomson scattering experiments [12], pulsed laser deposition [13], and photoacoustic microscopy [14]. This was presumably because of the lack of suitable laser sources that can operate in this mode, which is slowly changing in recent years. Until recently, burst-mode laser systems have relied on solid state lasers, with architectures that were not optimized for burst operation. In 2011, we demonstrated the first fiber laser designed to operate in the burst mode [15] and advanced it further with highly uniform intra-burst pulse energy distribution, enabled by advanced electronic synchronization and electronic signal burst preshaping [16]. This was followed by the demonstration of a rod-type fiber amplifier by Breitkopf et al. [17]. We have recently scaled the repetition rate to $1 \overline{\mathrm{MHz}}$ and average power to $100 \mathrm{~W}$ for material processing applications [18].

A burst of closely spaced pulses can have an effect similar to that of a single pulse of energy equal to that of the entire burst during its interaction with a given material [19]. In [20], Knappe et al. report a sixfold increase in material ablation rates with six lower energy pulses separated by $20 \mathrm{~ns}$, compared to a single pulse with energy equal to the sum of the burst. Particularly exciting opportunities abound in medical applications, where the possibility to utilize lower pulse energies to achieve a certain effect has major benefits in terms of lower heat deposition, reduced collateral damage, and eventually decreased possibility of medical complications. Marjoribanks and coworkers have recently reported the first results on burst-mode tissue processing $[21,22]$, which clearly demonstrate that the temporal properties of the burst envelope constitute effective 
additional degrees of freedom, which can be used to optimize material ablation while keeping thermal effects minimized. This can be understood by recalling that thermal effects depend predominantly on the average power or average fluence, while material ablation depends on the peak intensity. Thus, strength of the thermal effects can be controlled through controlling the burst repetition rate, while ablation efficiency can be kept high through the use of high repetition rate and high energy pulses inside the burst.

Given the great potential of burst-mode operation in future medical applications and material processing, which has already been implemented by various industrial systems, it is essential to understand the limitations on essential burst-mode parameters imposed by the laser physics. In particular, amplified spontaneous emission (ASE) generation is a major concern because of the burst repetition rate being typically in the $\mathrm{kHz}$ regime or even lower. Furthermore, because of the typically short durations of the burst, pumping cannot repopulate the upper state of the gain medium appreciably during the amplification of the pulse burst. Thus, the gain experienced by the pulse burst is almost entirely because of the pump absorbed prior to the arrival of the burst. Because of ASE, there is a limit to how early pumping can commence with respect to the arrival of the burst, which consequently limits, in practice, the amount of energy that can be stored. This limit depends also on the length of the gain medium. As a result, multiple trade-offs exist between keeping ASE low and burst energy high against keeping the gain medium sufficiently long for efficient pump absorption. Yet another concern is the desire to minimize the length of the gain fiber to minimize nonlinear effects.

In this contribution, we report the demonstration and detailed characterization of an all-fiber burst-mode $\mathrm{Yb}$ fiber amplifier with multi-stage (nine stages) amplification aiming to suppress ASE at a low repetition rate while employing a chirped fiber Bragg grating (CFBG) for a pulse stretcher with a stretching ratio of $\sim 1000$. The CFBG is designed to match an 1800 line $/ \mathrm{mm}$ transmission grating. This system is able to amplify 10-pulse bursts of $100 \mathrm{~ns}$ duration each to $400 \mu \mathrm{J}$ total energy. The individual pulses of $40 \mu \mathrm{J}$ average energy are compressed to $500 \mathrm{fs}$ with a 1800 line/mm grating compressor. The burst repetition rate is $1 \mathrm{kHz}$ and the intra-burst pulse repetition rate is $100 \mathrm{MHz}$ as determined by the seed oscillator. The amplifier system has a highly cascaded fiber integrated architecture designed to achieve high energy bursts made of high energy pulses at low burst repetition rates while suppressing ASE. Hence, ASE is carefully monitored at various stages and the output and pulsed pumping parameters are optimized to achieve the highest signal to ASE ratio. The gain performance and energy extraction limitations of the system are investigated for short duration bursts $(<500 \mathrm{~ns})$ desired for high per pulse energy.

\section{EXPERIMENTAL SETUP}

The experimental setup (Fig. 1) consists of an all-normal dispersion (ANDi) laser oscillator [23], seeding six stages of core pumped fiber preamplifiers, a three-stage double-clad (DC) fiber power amplifier, as well as synchronized pulse picking and pulsed pumping electronics. The overall, $\mathrm{Yb}$ all-fiber

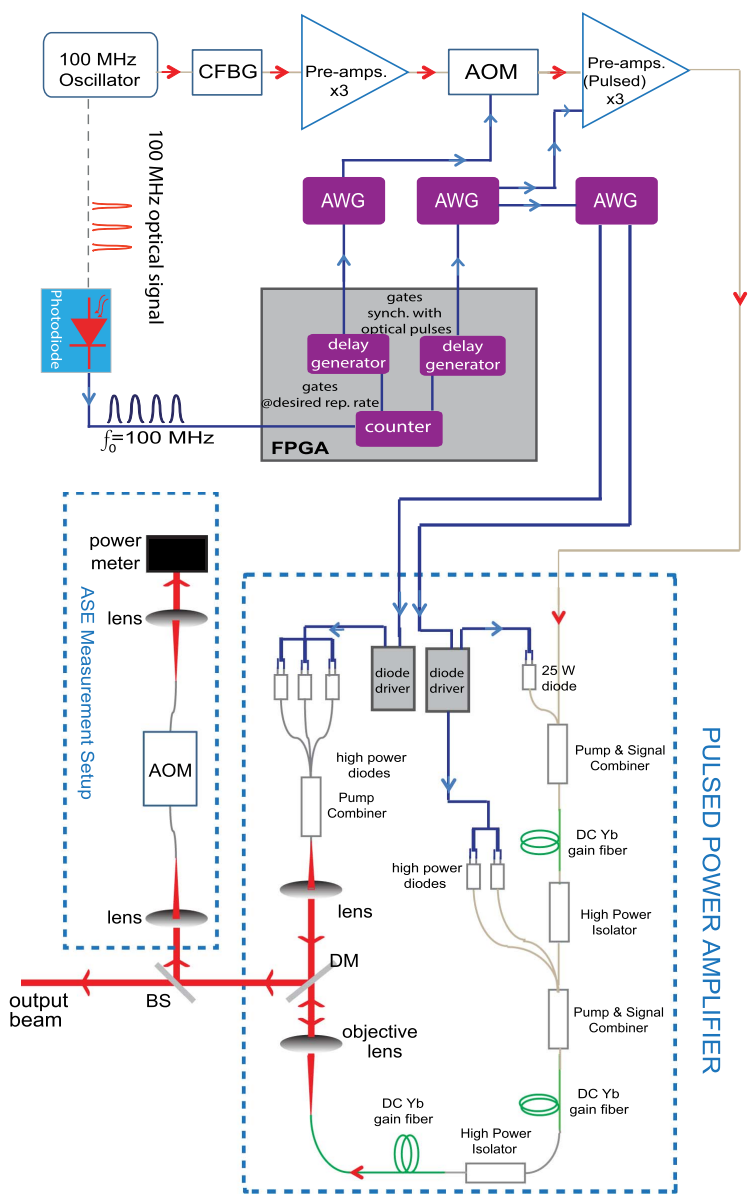

Fig. 1. Schematic diagram of the experimental setup. BS, beam sampler; DM, dichroic mirror; WDM, wavelength division multiplexer; PM, polarization maintaining; SM, single-mode; DC, double-clad.

master oscillator power amplifier architecture is based on the design in [24]. The first three stages of preamplifiers utilize continuous pumping while the latter three, which are positioned after the acousto-optic modulator (AOM), incorporate pulsed pumping. The $100 \mathrm{MHz}$ fiber oscillator outputs $\sim 130 \mathrm{~mW}$ with a spectrum $16 \mathrm{~nm}$ wide and centered at $1035 \mathrm{~nm}$ which is polarized by a polarization dependent isolator. From this point on, the system consists only of polarization maintaining (PM) components. The seed pulses are stretched to $2.3 \mathrm{~ns}$ in a CFBG, which replaced the stretch fiber which was increased to $2.6 \mathrm{~km}$ from $450 \mathrm{~m}$ to mitigate undesired nonlinear effects that occurred when the system was revised to its current multi-stage state. The CFBG is designed to match a compressor consisting of a pair of $1800 \mathrm{l} / \mathrm{mm}$ transmission gratings with a separation of $70 \mathrm{~cm}$ at an incidence angle of $69^{\circ}$. The seed signal drops down to $15 \mathrm{~mW}$ and the spectrum becomes $21 \mathrm{~nm}$ wide [inset of Fig. 2(d)] at the output of the pulse stretching unit made of a circulator and the CFBG. Thereafter, it is amplified by three stages of preamplifiers to nearly $1 \mathrm{~W}$ before entering the AOM. The preamplifiers are pumped continuously at $976 \mathrm{~nm}$, each with $500 \mathrm{~mW}$ and the length of the gain fiber in each preamplifier is chosen in a decreasing order $(85,60$, and $40 \mathrm{~cm})$ with the 
increasing signal at each stage such that the level of amplification is close to an optimum value. The AOM, which imprints the desired pulse burst envelope onto the signal, has a $2.3 \mathrm{~dB}$ insertion loss, $50 \mathrm{~dB}$ extinction, and rise and fall times of 6 and $8 \mathrm{~ns}$. At the output of the AOM, the signal power decreases to a mere $0.04 \mathrm{~mW}$ for 10 -pulse bursts repeated at $1 \mathrm{kHz}$, corresponding to $40 \mathrm{~nJ}$ of burst energy and an average energy of $4 \mathrm{~nJ}$ per pulse. Next, the bursts are amplified in six stages of pulsed amplification, for which the pump pulses need to be tightly synchronized with the seed signal to keep noise low. Comparatively short gain fibers are used in the three-stage pulsed preamplifier following the AOM to suppress ASE generation. The power amplifier at the end of the system also consists of three stages, the first two forward pumped and the final one backward pumped, which employ one, two, and three $25 \mathrm{~W}$ pump diodes, respectively. The three stages contain $\mathrm{Yb}-1200-\mathrm{DC}-20 / 125 \mathrm{PM}$ (nLight, Inc.) as gain fiber with lengths of $1.3,0.8$, and $0.8 \mathrm{~m}$ in respective order, each shorter than that required for high efficiency in continuous pumping regime. The details pertaining to the double-clad (DC) gain fiber, including the typical spatial mode quality can be found in our earlier work [25].

The pulse picking and the pulsed pumping processes are both synchronized using a signal derived from the $100 \mathrm{MHz}$ oscillator and managed by a field programmable gated array (FPGA) circuit. The FPGA circuit is triggered by the RF signal derived from the optical pulse train produced by the oscillator signal. This low jitter signal [26] is low-pass filtered to retain only the fundamental frequency of $100 \mathrm{MHz}$, which is used as the internal clock signal of the FPGA, ensuring the tightest possible synchronization allowed with the hardware limitations of an FPGA. The FPGA, in turn, triggers three arbitrary waveform generators (AWG), one of which drives the AOM and the other two providing pulses to diode drivers, which are capable of pulsed operation. The signal to the AOM is delayed with respect to the diode drivers by an adjustable amount. We set the value of this delay, such that the signal burst arrives precisely at the very end of the much longer pump pulse. The AWGs, in combination with the FPGA, allow us to adjust the durations of the pump pulses to maximize burst energy while suppressing ASE as much as possible. In addition, burst duration and the burst repetition frequency can be adjusted freely, although we are reporting here only results with 10 pulses within each burst.

\section{RESULTS AND DISCUSSION}

After optimization of all the signal durations and frequencies, the system was able to amplify $100 \mathrm{~ns}$ long 10 -pulse bursts to a net energy of $400 \mu \mathrm{J}$ at a burst repetition rate of $1 \mathrm{kHz}$ indicating an average per pulse energy of $40 \mu \mathrm{J}$. The temporal profile of the pulse train for $400 \mu \mathrm{J}$ bursts is shown in Fig. 2(a). The corresponding RF spectra recorded over a span of $50 \mathrm{MHz}$, centered at $100 \mathrm{MHz}$, show the RF comb generated by the burst system [lower panel of Fig. 2(b)]. Here, the $3 \mathrm{~dB}$ width of the main lobe is $10 \mathrm{MHz}$, which is consistent with the duration of the burst envelope (100 ns, in this case). Thus, the frequency domain representation of a train of pulse bursts constitutes a comb with $1 \mathrm{kHz}$ internal spacing, corresponding to the burst repetition frequency, under an envelope determined by the temporal shape of the burst envelope. Note that the comb lines are actually much denser than those displayed in
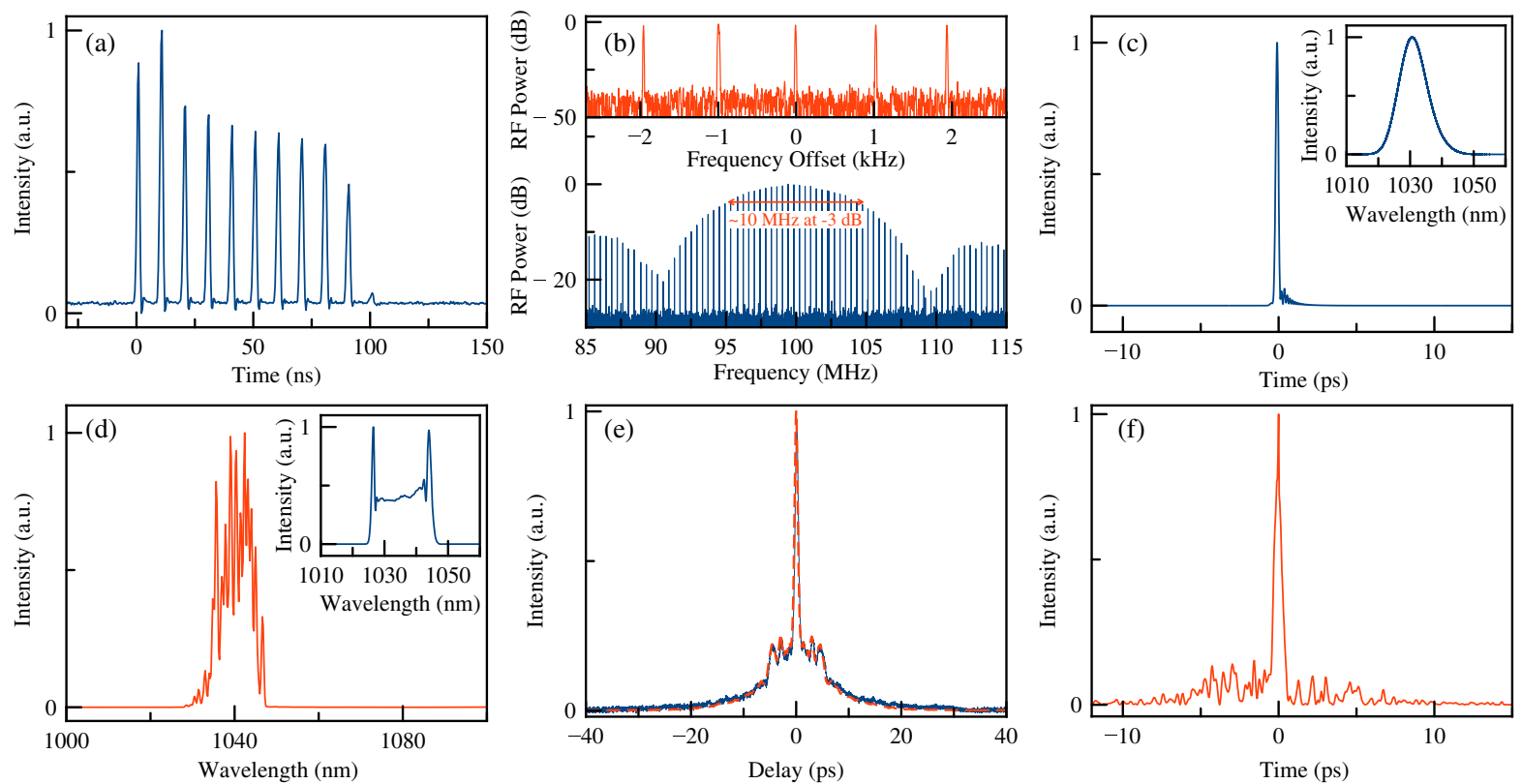

Fig. 2. (a) Measured temporal profile of the $100 \mathrm{~ns}$ pulse burst, which repeated at $1 \mathrm{kHz}$. (b) Measured RF spectrum of the pulse train in burst mode. Lower panel shows a frequency span of $20 \mathrm{MHz}$ centered at $100 \mathrm{MHz}$. Top panel: Close-up showing the $1 \mathrm{kHz}$ lines with a span of $5 \mathrm{kHz}$ versus frequency offset from the central frequency of $100 \mathrm{MHz}$. (c) Pulse shape obtained for $40 \mu \mathrm{J}$ pulse energy, using pulse propagation simulations. Inset: Simulated output spectrum. (d) Measured optical spectrum for $40 \mu \mathrm{J}$ pulse energy. Inset: Spectrum at the output of CFBG, which seeds the amplifier chain. (e) Measured (solid curve) and retrieved (dashed curve) autocorrelation traces of the dechirped pulses at the $40 \mu \mathrm{J}$ energy level. (f) Retrieved temporal profile of the pulse using the PICASO algorithm based on the measured autocorrelation and spectrum. 
the figure, which is because of the finite number of sample points that the spectrum analyzer (FSUP26, Rohde \& Schwarz) can record. A close-up of the same signal with a span of $100 \mathrm{kHz}$ and resolution bandwidth of $10 \mathrm{~Hz}$ correctly reveals this fine comb structure with $1 \mathrm{kHz}$ spacing between the lines [top panel of Fig. 2(b)].

Control of nonlinearity is a major challenge in a high-energy, multi-stage integrated fiber amplifier system where pulse propagation simulations $[\underline{25}, 27]$ guided us in determining the extent of required pulse stretching and attainable pulse energies. Our main criterion was to suppress nonlinearity so that self-phase modulation (SPM) and Raman scattering are reduced to acceptable levels, such that pulse compression below picosecond level is possible. This task is intricately coupled to optimization of gain levels and minimization of ASE, as explained below. One technical limitation was the size of gratings commercially available for pulse compression. In practice, the $20 \mathrm{~cm}$ width of the 1800 line $/ \mathrm{mm}$ transmission gratings that we could obtain sets the upper limit for pulse stretching, given a certain spectral width. For the spectra reported here, the gratings allowed stretching up to 2.3 ns near Litrow configuration with a separation of about $70 \mathrm{~cm}$. At this setting, the third-order dispersion (TOD) of $\sim 1.4 \mathrm{ps}^{3}$ imposed by the gratings, if not compensated for, renders pulse compression below 10 ps impossible. A CFBG was utilized as it does not detract from the fiber integrated structure of the system and allows compensation of the TOD from the grating compressor. Simulation results demonstrated that, with a CFBG that matched the grating compressor with the opposite sign for TOD, a duration around $250 \mathrm{fs}$ for compression of $40 \mu \mathrm{J}$ pulses was obtainable [Fig. 2(c) for temporal profile and inset of Fig. 2(c) for the spectral profile]. Hence, a CFBG was integrated to the system at the output of the oscillator stretching pulses with a $21 \mathrm{~nm}$ wide spectrum up to $2.3 \mathrm{~ns}$.

Guided by the simulation results, the multiple stages of amplification were optimized to minimize ASE, while achieving a balance between burst energy and pulse compressibility, as limited by SPM. The ASE optimization is described in detail below. The measured optical spectrum is shown in Fig. 2(d), where the inset shows the seed spectrum. The nonlinear phase shift was estimated around $4 \pi$ for the power amplifier section when amplified pulse energy reaches $40 \mu \mathrm{J}$ at output. The temporal profiles of the amplified pulses with energy of $40 \mu \mathrm{J}$ were characterized after compression with a long-range autocorrelator [Fig. 2(e)], while the system was operated at uniform repetition rate. Finally, we used the PICASO algorithm [28] to retrieve an estimate of the pulse form [Fig. 2(f)]. It should be mentioned that, for a complex pulse shape as this, the PICASO algorithm cannot be expected to give a completely reliable temporal profile. However, it is successful in matching the measured autocorrelation result with high precision as evidenced by the red dashed curve in Fig. 2(f). Thus, it should be interpreted as a good indicator of the size of the pedestal and the effective pulse width. The retrieved pulse width (full width at half-maximum, FWHM) was $500 \mathrm{fs}$. This is longer than the simulation results by a factor of two. Part of this discrepancy because of the experimental spectrum being $10 \%-15 \%$ narrower than the simulated one. However, we attribute the main source of deviation to the highly structured experimental spec- trum. This structure is mostly coming from a small amount of power being coupled to the orthogonal polarization mode or a higher spatial mode at different points along the nine stages of amplification. When small amounts of power (much less than $\% 1$ ) are coupled into another mode, which would have a slightly different group velocity, the main pulse and the small replica of the pulse co-propagate while maintaining almost complete temporal overlap since the pulses are chirped to 2.3 ns. Numerical simulations indicate that, during amplification of highly chirped pulses in the presence of strong SPM, energy can be transferred from the main pulse to the secondary pulses, which, in turn, can create a complex temporal structure as in Fig. 2(f). This interesting, but limiting, phenomenon will be investigated further in the future and reported elsewhere.

The main limitation to burst energy arises from ASE generation in an effectively low repetition system like ours. Since ASE generation can easily be overlooked, it was investigated diligently together with gain performance in the stages which employed pulsed pumping. At first, the output of the threestage pulsed preamplifier was studied to obtain maximum burst energy while suppressing the ASE level. Varying the duration of the pump pulse applied at each stage while keeping the peak pump power at or close to the maximum level of $\sim 540 \mathrm{~mW}$, pumping the first two stages with a lower energy level, and increasing it for the third stage resulted in the best combination of high burst energy and low ASE content. Thereby, a net (free of ASE) signal power of $16 \mathrm{~mW}$ (burst energy of $16 \mu \mathrm{J}$ ) with an ASE content of 3\% was obtained. All together, the three-stage, core-pumped preamplifier could amplify $100 \mathrm{~ns}$ bursts repeated at $1 \mathrm{kHz}$ with a net signal gain of $25 \mathrm{~dB}$ and a pump-to-signal conversion of $16 \%$, while limiting the ASE to signal ratio to less than $5 \%$. The results indicate a threshold pump energy of $95 \mu \mathrm{J}$ for ASE generation. When compared with the performance of the single pulsed preamplifier (threshold of $16 \mu \mathrm{J}$, conversion of $2.5 \%$ ) in the previous version of the system [15], a sixfold increase, both in threshold pump energy for ASE onset and pump-to-signal conversion efficiency for similar ASE level has been achieved via three-stage preamplification.

Having explored the optimum parameters for the pulsed preamplifier, we next investigated the power amplifier. The power amplifier consists of three stages, where the first and second stages are forward pumped by one and two $25 \mathrm{~W}$ diodes, respectively, while the final stage is backward pumped by three $25 \mathrm{~W}$ diodes. The performance at the output of each stage was analyzed. For the first two stages, identical drive signal is used which generates a pump pulse for the second stage that is temporally equal, but with twice the amplitude of that of the first stage. Thus, twice as much peak pump power and pump energy are applied to the second stage. In respect of this fact, the results for the first stage will be discussed together with the second one which represents the cumulative amplification of the two stages. This will be followed by the analysis of the final backward pumped stage driven with a pump pulse independent of the first two stages, where the input signal is taken as the output of the second stage.

To study the performance at the output of the first and second stages of the power amplifier, the input burst energy to the 
power amplifier was set to $10 \mu \mathrm{J}$ with ASE level near zero. The burst energy was reduced down from $16 \mu \mathrm{J}$ at the output of the pulsed preamplifier because of losses introduced by two high power PM isolators and the monitoring port located between the isolators. The near zero ASE content resulted from effective filtering of the isolators. This was critical since even a very small ASE content at input degrades the net signal amplification significantly by stealing the gain. The pump diodes were driven with pulses of $40,50,60$, and $70 \mu$ s durations and $60 \%, 75 \%$, and $100 \%$ of full power level. The net burst energy, total output, and ASE energy versus the pump energy are shown in Figs. 3(a) and 3(b), for the output of the first and second stages, respectively. The results demonstrate a similar trend of converging amplified burst energy $(230 \mu \mathrm{J}$ for the first and $290 \mu \mathrm{J}$ for the second one) and threshold behavior for ASE onset with increasing pump energy. The limit for net amplified burst energy shifts to a higher level for the two-stage amplification. Correspondingly, the same burst energy is obtained with a significantly less ASE level $(220 \mu \mathrm{J}$ with $17 \%$ compared to $32 \%$ of first stage), and the threshold pump energy for ASE generation doubles from $\sim 0.5 \mathrm{~mJ}$ for the first stage to $\sim 1 \mathrm{~mJ}$ for the second one, demonstrating the ASE suppression effect of cascaded amplification. Figure 3(d) shows the fractional ASE content in the output of each stage which grows nearly linearly above the threshold indicating an exponential growth. The pump-to-signal conversion ratio [Fig. 3(e)], a critical parameter for a system based on energy storage, increases from below $10 \%$ with pump energy up to a maximum of $15 \%$ whereafter it degrades because of ASE taking over at output of both stage 1 and 2. However, the total output [Figs. $\underline{3(\mathrm{a})}$ and $\underline{3(\mathrm{~b})}$ ] grows fairly linearly, with a slope of $45 \%$ and $30 \%$, for the two-stage and single-stage amplification, respectively, indicating operation in the unsaturated regime.

Next, pulsed pumping performance was measured at the output of the final backward pumped stage with prior pulsed amplification stages set to produce an input with a near zero ASE level. Figure 3(c) shows the amplification results for a net input burst energy of $50 \mu \mathrm{J}$. The pump to signal conversion efficiency and amplified burst energy follow a similar trend with the preceding stages but both at a higher level. The conversion increases from $14 \%$ to $22 \%$ and degrades afterward [Fig. 3(e)] while the burst energy converges a little above $400 \mu \mathrm{J}$. ASE is generated above a threshold pump energy level near $1 \mathrm{~mJ}$ similar to the two-stage amplification case [Figs. 3(c) and 3(d)]. Overall, the results for the power amplifier indicate that there is a pump energy level for which the pump-to-signal conversion reaches a maximum and afterward starts decaying as any further increase in stored energy is converted to ASE. Further, measurements were also taken with signal input containing ASE and even just a few percent ASE caused a significant drop in the pump to signal conversion efficiency while the ASE growth was enhanced. For example, at pump energy of $1.5 \mathrm{~mJ}$ in the final stage, an input signal burst of $66 \mu \mathrm{J}$ and $3 \%$ ASE is amplified to a net energy of $380 \mu \mathrm{J}$ with $38 \%$ ASE content and $21 \%$ pump-to-signal conversion, while $190 \mu \mathrm{J}$ and $11 \%$ ASE is amplified to $320 \mu \mathrm{J}$ with an ASE of $56 \%$ and conversion of only $11 \%$.

To shed light on the temporal dynamics of ASE buildup, ASE generation was temporally characterized at the output of the system [Fig. $\underline{3(\mathrm{f})]}$, for the case of a pump pulse of
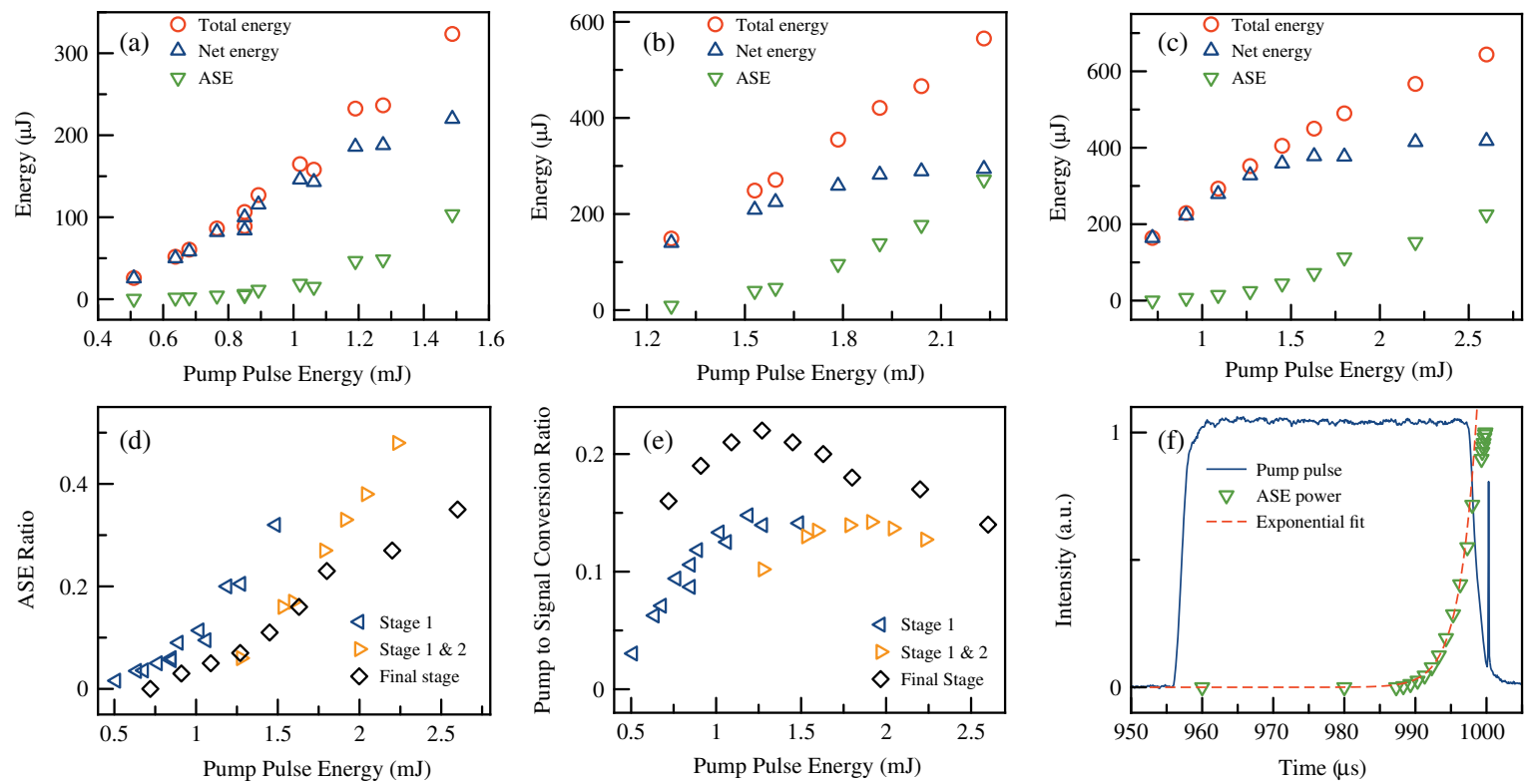

Fig. 3. Measured net amplified signal (triangles facing up), ASE (triangles facing down) and total (circles) output energy at output of the (a) first stage of power amplifier, (b) second stage of power amplifier, and (c) final stage of power amplifier, as a function of the pump pulse energy. (d) ASE fraction in output for each stage. (e) Pump-to-signal conversion efficiency at the output of each stage. Note that in (d) and (e), the data for the second stage output is designated by "Stage 1 and 2" to indicate the cumulative amplification. (f) Temporal characterization of ASE generation in the case of $1.7 \mathrm{~mJ}$ pump pulse with $40 \mu$ s duration, amplified net burst energy of $400 \mu \mathrm{J}$ and $20 \%$ ASE ratio in the output. ASE generation (triangles facing down) versus time and exponential fit (dashed line) to it with the formula of $I(t)=0.017 \times e^{(t-989) / 2.43}$. Pump and signal pulses are also shown (solid line). The arrival of the signal burst at $1 \mathrm{~ms}$ mark can also be seen. 
$1.7 \mathrm{~mJ}$ and $40 \mu$ s duration, amplified net burst energy of $400 \mu \mathrm{J}$, and $20 \%$ ASE ratio in the output. The temporal growth profile is fit well by an exponential, until the ASE growth also starts to saturate because of pump depletion. The time constant is $2.4 \mu$ s over a period of $\sim 10 \mu$ s before the signal burst arrives and terminates once the signal burst arrives at the $1 \mathrm{~ms}$ mark. Since the launched signal has no ASE content, the ASE growth initiating $\sim 30 \mu \mathrm{s}$ after the onset of the pump pulse indicates a threshold of $\sim 1 \mathrm{~mJ}$ stored pump energy for the parameters considered here in agreement with Figs. 3(c) and 3(d).

The exponential growth of ASE setting off above a threshold pump energy limits the pump-to-signal conversion. The critical question is whether there is a way to increase this limit and extract significantly higher levels of burst energy from fiber amplifiers for short duration ( $<500 \mathrm{~ns}$ ) bursts. One way of attacking this problem is to increase the peak pump power so that the same pump energy can be stored in a shorter period, allowing less time for ASE buildup. This was tested in the power amplifier by applying the same pump energy with two different pulses, one of them twice as long as the other, in two different locations: first at the second stage and second in the backward pumped final stage. In the first case, the amplified signal for $1.3 \mathrm{~mJ}$ pump energy was compared for 20 and $40 \mu$ s pulse duration, and longer pulse yielded $\sim 50 \%$ higher net signal output with nearly two times the ASE-to-signal ratio. In the second case of backward amplifier, $1.3 \mathrm{~mJ}$ of pump energy with durations of 30 and $60 \mu \mathrm{s}$, and $0.9 \mathrm{~mJ}$ with durations of 42 and $21 \mu$ s were tested and, in both cases, the shorter pump pulse with higher peak power did not produce a noteworthy improvement, either in the signal-to-ASE ratio or in the pump-to-signal conversion. It may be necessary to increase the peak pump power to even higher levels and use pump pulse durations close to the $10 \mu$ s level or less, to really test the effect of shorter pump pulses on ASE suppression, which would require boosting the current pump capacity of our system significantly. Increasing gain fiber lengths, in addition to using such high peak power pump pulses, may also boost the amplified burst energy by increasing pump energy storage and thereby signal gain. However, this also increases the possibility of giant pulse formation that can damage the system, and further increase detrimental nonlinear effects. Note that the system is close to the limits of pulse stretching because of the commercially available largest compressor currently in use, and cannot tolerate a substantial increase in nonlinearity. For the same reason, the obvious alternative of increasing the number of amplifier stages is not really viable, even before considering the undesirable effects on the spectrum which are mentioned above in the discussion on pulse compression results. Yet, another possibility of achieving a higher level of burst energy at the output is by placing an AOM at the input of the final stage of the power amplifier and maximizing the input signal burst energy to this stage, while eliminating ASE that comes with it using the AOM. This way, energy extraction limit is not actually raised in any of the stages, but cumulative amplification can be boosted by relaxing the near zero ASE enforcement at the input of the final stage which limits the signal burst energy here and, thereby, the amplified signal at the output. Considering the amplification results at the output of the second and final stages, an increase in the $200-300 \mu \mathrm{J}$ range can be expected in the output burst energy that is as long as nonlinear effects allow. However, the large-mode DC fibers used in the power amplifier may require a free-space AOM for ASE elimination, which will impair the all-fiber architecture of the system, as well as increase complexity. Finally, more than 30 -fold improvement in the ASE threshold obtained with $\mathrm{Yb} 1200$ 20/125 DC fiber in the single stage forward pumped amplification, compared to the single-mode single stage preamplifier, points to the energy storage capacity of the fiber. The increase in the threshold is in the same order with the ratio $(\sim 40)$ of total active ion population of the $\mathrm{DC} \mathrm{Yb}$ fiber used to that of the single mode one. Note that it is the energy storage capacity which is a function of total active ion population that seemingly raises the ASE threshold, not the gain which is a function of active ion concentration. In fact, the gain enhances ASE generation and it is lowered by using relatively short active fibers to suppress ASE in the pulsed amplification stages of our system. Accordingly, gain fibers with larger core and pump cladding dimension that can store higher levels of pump energy might have the potential to raise the ASE threshold and enable higher burst energy extraction, as long as nonlinear effects can be kept under control. However, it should be mentioned that such fibers with larger cross sections may cause adversities, such as reduced output beam quality which is currently close to an $M^{2}$ of 1 in our system and technical difficulties because of splicing complications.

\section{CONCLUSION}

In conclusion, we have demonstrated an all-fiber laser system centered at $1040 \mathrm{~nm}$, generating 10-pulse bursts with $400 \mu \mathrm{J}$ energy at a $1 \mathrm{kHz}$ repetition rate. The average energy per pulse is $40 \mu \mathrm{J}$ and pulse-to-pulse separation is $10 \mathrm{~ns}$. By incorporating a CFBG with dispersion matching that of the grating compressor, the pulses could be compressed to $500 \mathrm{fs}$. We have characterized and minimized ASE generation, while keeping a reasonably high burst energy and low fiber nonlinearity at the cost of relatively reduced pumping efficiency. We consider this to be a worthwhile trade-off because of low complexity and cost of laser diodes that can be used to pump Yb-fiber lasers. ASE generation, which is the main limitation to burst energy has been characterized in detail, and the presence of a threshold pump energy for ASE generation was established as a characteristic of pulsed pumping regime. Possibilities for further improvements are discussed which involve applying pump pulses with considerably higher peak power and short duration comparable to ASE buildup period, maximizing input signal energy to the final stage while eliminating ASE via an AOM before that stage, and finally using gain fibers with larger cross sections with higher pump energy storage capacity.

TÜBITAK (112T980); European Research Council (ERC) (ERC-617521 NLL).

F. Ö. I. acknowledges support from the European Research Council (ERC) Consolidator Grant ERC-617521 NLL. 


\section{REFERENCES}

1. D. J. Richardson, J. Nilsson, and W. A. Clarkson, "High power fiber lasers: current status and future perspectives [Invited]," J. Opt. Soc. Am. B 27, B63-B92 (2010).

2. J. Nilsson and D. N. Payne, "High-power fiber lasers," Science 332, 921-922 (2011).

3. M. E. Fermann and I. Hartl, "Ultrafast fibre lasers," Nat. Photonics 7, 868-874 (2013).

4. C. Jauregui, J. Limpert, and A. Tünnermann, "High-power fibre lasers," Nat. Photonics 7, 861-867 (2013).

5. K. Sugioka and Y. Cheng, Ultrafast Laser Processing: from Micro- to Nanoscale (CRC Press, 2013).

6. S. H. Chung and E. Mazur, "Surgical applications of femtosecond lasers," J. Biophotonics 2, 557-572 (2009).

7. M. Lapczyna, K. P. Chen, P. R. Herman, H. W. Tan, and R. S. Marjoribanks, "Ultra high repetition rate $(133 \mathrm{MHz})$ laser ablation of aluminum with 1.2-ps pulses," Appl. Phys. A 69, S883 (1999).

8. H. Braun, R. Corsini, J. Delahaye, A. de Roeck, S. Döebert, A. Ferrari, G. Geschonke, A. Grudiev, C. Hauviller, B. Jeanneret, E. Jensen, T. Lefevre, Y. Papaphilippou, G. Riddone, L. Rinolfi, W. D. Schlatter, H. Schmickler, D. Schulte, I. Syratchev, M. Taborelli, F. Tecker, R. Toms, S. Weisz, and W. Wuensch, "CLIC 2008 Parameters," CERN-OPEN2008-021 (2008)

9. I. Will, H. I. Templin, S. Schreiber, and W. Sandner, "Photoinjector drive laser of the FLASH FEL," Opt. Express 19, 23770-23781 (2011).

10. P. Wu, W. R. Lempert, and R. B. Miles, "Megahertz pulse-burst laser system and visualization of shock-wave/boundary-layer interaction," AIAA J. 38, 672-679 (2000).

11. B. S. Thurow, A. Satija, and K. Lynch, "Third-generation megahertzrate pulse burst laser system," Appl. Opt. 48, 2086-2093 (2009).

12. D. J. Den Hartog, J. R. Ambuel, M. T. Borchardt, A. F. Falkowski, W. S. Harris, D. J. Holly, E. Parke, J. A. Reusch, P. E. Robl, H. D. Stephens, and Y. M. Yang, "Pulse-burst laser systems for fast Thomson scattering (invited)," Rev. Sci. Instrum. 81, 10D513 (2010).

13. M. Murakami, B. Liu, Z. Hu, Z. Liu, Y. Uehara, and Y. Che, "Burstmode femtosecond pulsed laser deposition for control of thin film morphology and material ablation," Appl. Phys. Express 2, 042501 (2009).

14. T. Liu, J. Wang, G. I. Petrov, V. V. Yakovlev, and H. F. Zhang, "Photoacoustic generation by multiple picosecond pulse excitation," Med. Phys. 37, 1518-1521 (2010).

15. H. Kalaycioglu, K. Eken, and F. Ö. Ilday, "Fiber amplification of pulse bursts up to $20 \mu \mathrm{J}$ pulse energy at $1 \mathrm{kHz}$ repetition rate," Opt. Lett. 36, 3383-3385 (2011).
16. H. Kalaycıoğlu, Y. B. Eldeniz, Ö. Akcaalan, S. Yavas, K. Gurel, M. Efe, and F. Ö. Ilday, "1-mJ pulse bursts from a Yb-doped fiber amplifier," Opt. Lett. 37, 2586-2588 (2012).

17. S. Breitkopf, A. Klenke, T. Gottschall, H. J. Otto, C. Jauregui, J. Limpert, and A. Tunnermann, " $58 \mathrm{~mJ}$ burst comprising ultrashort pulses with homogenous energy level from an Yb-doped fiber amplifier," Opt. Lett. 37, 5169-5171 (2012).

18. P. Elahi, S. Yilmaz, Y. B. Eldeniz, and F. Ö. Ilday, "Generation of picosecond pulses directly from a $100 \mathrm{~W}$, burst-mode, doping-managed Yb-doped fiber amplifier," Opt. Lett. 39, 236-239 (2014).

19. W. Hu, Y. C. Shin, and G. King, "Modeling of multi-burst mode picosecond laser ablation for improved material removal rate," Appl. Phys. A 98, 407-415 (2010).

20. R. Knappe, H. Haloui, A. Seifert, A. Weis, and A. Nebel, "Scaling ablation rates for picosecond lasers using burst micromachining," Proc. SPIE 7585, 7850H (2010).

21. R. S. Marjoribanks, C. Dille, J. E. Schoenly, L. McKinney, A. Mordovanakis, P. Kaifosh, P. Forrester, Z. Qian, A. Covarrubias, Y. Feng, and L. Lilge, "Ablation and thermal effects in treatment of hard and soft materials and biotissues using ultrafast-laser pulse-train bursts," Photon. Lasers Med. 1, 155-169 (2012).

22. Z. Qian, A. Mordovanakis, J. E. Schoenly, A. Covarrubias, Y. Feng, L. Lilge, and R. S. Marjoribanks, "Pulsetrain-burst mode, ultrafast-laser interactions with $3 \mathrm{D}$ viable cell cultures as a model for soft biological tissues," Biomed. Opt. Express 5, 208-222 (2014).

23. A. Chong, J. Buckley, W. Renninger, and F. W. Wise, "All-normaldispersion femtosecond fiber laser," Opt. Express 14, 1009510100 (2006).

24. F. Ö. Ilday, H. Lim, J. Buckley, and F. W. Wise, "Practical, all-fiber source of high-power, 120-fs pulses at 1 micron," Opt. Lett. 28, 1362-1364 (2003).

25. P. K. Mukhopadhyay, K. Özgören, I. L. Budunoglu, and F. Ö. Ilday, "All-fiber low-noise high-power femtosecond Yb-fiber amplifier system seeded by an all-normal dispersion fiber oscillator," IEEE J. Sel. Top. Quantum Electron. 15, 145-152 (2009).

26. A. Winter, F. Ö. Ilday, O. D. Mücke, R. Ell, H. Schlarb, P. Schmüser, and F. X. Kärtner, "Towards high-performance optical master oscillators for energy recovery linacs," Nucl. Instrum. Methods A 557, 299-304 (2006).

27. B. Oktem, C. Ülgüdür, and F. Ö. Ilday, "Soliton-similariton fibre laser," Nat. Photonics 4, 307-311 (2010).

28. J. W. Nicholson, J. Jasapara, W. Rudolph, F. G. Omenetto, and A. J. Taylor, "Full-field characterization of femtosecond pulses by spectrum and cross-correlation measurements," Opt. Lett. 24, 1774-1776 (1999). 